\title{
Apuntes teóricos para historiar los procesos educativos fuera del espacio escolar. Reflexiones desde el noreste de México
}

Theoretical Reflections on the History of Educational Processes Beyond the School. A Perspective from the Mexican Northeast

\author{
Mónica Lizbeth Chávez González \\ Escuela Nacional de Estudios Superiores, \\ Unidad Morelia, UNAM, \\ mochago@hotmail.com
}

\begin{abstract}
Resumen
Este artículo presenta reflexiones teóricas en torno a la educación, con el fin de construir rutas analíticas que permitan abordar históricamente este fenómeno, más allá de la dinámica institucional. Estas reflexiones surgen en el marco de un proyecto específico cuyo objetivo central es analizar las prácticas y los conocimientos educativos en el sur de Nuevo León, desde una mirada sociocultural y etnohistórica. En el proceso de construcción de la región de estudio he identificado problemáticas específicas en la zona que requieren un tratamiento teórico particular, el cual he ordenado a partir de tres ejes específicos: el papel que cumple la socialización en el campo educativo, los aportes del enfoque intercultural, especialmente en el análisis de procesos culturales e identitarios y, finalmente, la necesidad de reflexionar sobre los "aprendizajes para la vida" para ampliar y complejizar el análisis histórico de la educación. La intención final de este artículo no es presentar un corpus teórico concluido, sino exponer, para su discusión, las primeras reflexiones sobre cómo se abordarían los procesos educativos, más allá del espacio prescriptivo e institucionalizado que representa la escuela.
\end{abstract}

Palabras claves: educación, socialización, interculturalidad, aprendizajes para la vida. 


\begin{abstract}
This article offers a theoretical discussion aimed at the development of analytical perspectives for a history of education beyond that of institutionalized spaces. It arises from a project studying educational practice and knowledge in the south of the state of Nuevo León (Mexico) from a sociocultural and ethnohistorical perspective. During the definition and delimitation of the region of study various problems demanding theoretical treatment were identified and organized around three axis: the role of socialization in the educational field, the contributions of an intercultural perspective to the study of cultural and identity processes and, thirdly, the need to reflect on the importance of "learning for life" in order to widen the historical analysis of education and make it more complex. The final objective of the article is not to present a theoretical corpus but to outline for future discussion some theoretical points suggesting how to study educational processes beyond the prescriptive and institutionalized space of the school.
\end{abstract}

Abstract

Keywords: Education, Socialization, Interculturality, Learning for Life.

\title{
Introducción
}

Hasta hace unas décadas, la historiografía de la educación en México transitó de las políticas institucionales hacia el estudio de las prácticas cotidianas, los discursos y representaciones, la circulación de objetos y disciplinas escolares, los métodos de enseñanza, las tácticas disciplinarias, los sistemas de exámenes, la conformación del tiempo y el espacio, además de hacer visibles las experiencias de individuos poco estudiados, como los niños, los indígenas o las maestras (Galván et al., 2003). A partir de los años noventa, esta historia escrita comenzó a "documentar lo no documentado", o a revisar los silencios y ausencias dentro del espacio escolar. Estos temas se incluyeron gracias al tratamiento de nuevas fuentes de investigación, como las fotografías, los objetos, la literatura y libros de texto, así como por el uso de la etnografía escolar. Dentro de esta corriente historiográfica se abrió una amplia gama de interrogantes sobre temas poco estudiados, aunque se ha seguido privilegiando la dinámica escolar, soslayando otros conocimientos, escenarios y dinámicas fundamentales para la formación social de los sujetos que escapan de lo prescriptivo y que, sin duda, tienen un peso en la formación de las subjetividades e identidades sociales. Esta ausencia se debe, en parte, a la falta de diálogo interdisciplinario que permita problematizar y enriquecer teórica y metodológicamente los alcances de la educación en un sentido más amplio.

De algunos años a la fecha, algunos historiadores de la región se han preocupado por realizar investigaciones originales desde perspectivas teóricas y metodológicas novedosas, en las que resalten las particularidades de este espacio de estudio. La mirada regionalista ha 
permitido romper con la visión centralista y homogeneizadora de la historiografía política de la educación para abrir paso al conocimiento de procesos diversos y complejos en la región noreste respecto de las dinámicas nacionales. Por ejemplo, se ha señalado la fuerte influencia que ejerció la pedagogía estadounidense sobre la francesa a finales del siglo XIX (Ramos, 2011), el auge de la educación técnica e industrial sobre la profesional (Garza, 2010), la poca influencia de la doctrina socialista en el campo político y educativo (Garza, 2010; Espinosa, 2006), el arribo tardío de una educación bilingüe por la imagen difundida de un "norte sin indígenas" (López, 2010; Chávez, 2010), la importancia de la migración masculina y de su fuerte ingreso al trabajo agroindustrial en la pronta feminización del magisterio (Ramos, 2006), entre otros.

Siguiendo este interés por ubicar la producción de conocimiento histórico sobre la educación, desde un diálogo interdisciplinario y desde las particularidades que las diferentes regiones nos ofrecen para debatir teórica y metodológicamente los hallazgos en este campo, nos proponemos reconsiderar algunos apuntes teóricos sobre el tema educativo. Estas reflexiones surgen en el marco de un proyecto específico cuyo objetivo central es analizar las prácticas y los conocimientos educativos en el sur de Nuevo León, bajo una óptica sociocultural y etnohistórica.

En el proceso de construcción de la región de estudio he identificado problemáticas específicas en la zona que requieren un tratamiento teórico particular, el cual presento a partir de tres ejes específicos: el papel de la socialización en el campo educativo, los aportes del enfoque intercultural (especialmente en el estudio de los procesos culturales e identitarios) y, finalmente, la necesidad de reflexionar sobre los "aprendizajes para la vida", de tal modo que se amplíe y complejice el análisis histórico de la educación. Si bien los temas de este artículo surgen de un contexto regional específico, su discusión resultaría de interés para estudios de otras regiones. Por ello, en las línes subsiguientes, en lugar de desarrollar las características de la región, me centraré en los aspectos conceptuales y metodológicos de los tres ejes mencionados.

\section{La educación como proceso de socialización}

Estudiar los procesos educativos desde el enfoque de la socialización planteada por los antropólogos cognitivos nos permite interesarnos por la forma en que el mundo se construye socialmente y es interiorizado por los individuos, a través de varios mecanismos, espacios e intensidades. Implica reconocer que la educación es, en un sentido amplio, un entramado de aprendizajes enmarcados en diversos contextos, en los cuales un sujeto conoce el mundo que lo rodea y lo aprehende como suyo para conformarse en un ser social. Con ello se ubica a la escuela como uno de los tantos campos de interacción en los que un 
individuo se educa y se pone especial acento en las prácticas, las representaciones, las experiencias concretas y los aprendizajes significativos, adquiridos más allá del espacio institucionalizado. Vista así la educación, se sobreentiende que el foco de interés está fuera de los fines pedagógicos y didácticos que persigue la escuela, para observar, en cambio, los procesos y espacios que conducen al aprendizaje de comportamientos, valores, representaciones y significados sociales.

El concepto de socialización no es nuevo en la teoría antropológica ni en la sociología educativa, pues tiene al menos cuatro décadas de haberse introducido, especialmente a partir del texto clásico de Berger y Luckman (1979). Desde entonces, la sociología cognitiva ha adquirido más fuerza y se ha difundido a otras disciplinas, aunque es evidente que los niveles de influencia no han sido los mismos, no al menos en el caso de la historiografía educativa. En las últimas décadas, los enfoques en torno a la socialización han transitado de posturas estructuralistas basadas en la interiorización de normas y comportamientos socialmente validados, hacia otras que resaltan la capacidad de agencia de los sujetos, quienes reelaboran los modelos sociales continuamente. Ambas posturas parecen conciliarse si consideramos que la socialización alude tanto al proceso de interiorización de las normas, valores y pautas culturales necesarias para la vida en sociedad, dentro de un tiempo y espacio específico (Berger y Luckman, 1979; Goslin, 1969; Rhys, 1972; Whiting et al., 1966), como para el proceso mediante el cual los individuos desarrollan una capacidad de adaptación a un entorno múltiple y cambiante que les ofrece diversas experiencias significativas para constituirse como sujetos (Lewis y Feinman, 1991; Dubet y Martucelli, 1998). El concepto permite entender cómo las estructuras sociales son apropiadas por el sujeto y también cómo el sujeto va reelaborando continuamente las experiencias que le ofrecen estas estructuras; toma posturas y decisiones con cierta autonomía sobre el mundo que se le presenta. Así, se llega a complementar la perspectiva clásica, la cual resulta útil para no perder de vista los factores estructurales presentes en las decisiones de los individuos, para considerar la dimensión del poder de las estructuras y entender cuáles son los límites de la acción individual, que no siempre es totalmente autónoma ni libre.

Por su parte, las corrientes centradas en la agencia suelen omitir la manera en que el orden social se transmite para centrarse en cómo ocurre el proceso de internalización a nivel individual, a partir de experiencias concretas. Para estos últimos autores el individuo es un actor de su propia socialización y no entienden la personalidad como una especie de modelo social internalizado.

En la vida diaria, el individuo interactúa con otros y adquiere el conocimiento social acumulado históricamente para percibir la realidad como un mundo coherente y construir un "yo individual" y un "yo colectivo". El mecanismo a través del cual el mundo social se proyecta en la conciencia de un individuo y orienta sus actos es la internalización (Berger y Luckman, 1979). 
Por medio de la socialización un individuo moldea sus percepciones sobre la sociedad y se posiciona en ésta, a la vez que es posicionado dentro de la misma. Las experiencias que se adquieren, sin embargo, no son iguales en todos los escenarios ni momentos de interacción.

En la actualidad, el mundo no es accesible como un todo más o menos estable y organizado, sino que se concibe o entiende a partir de una diversidad de esquemas culturales e ideológicos que complejizan la identificación del sujeto. Los individuos generan experiencias que les permiten integrarse a la sociedad con tensiones y conflictos, bajo la intermediación de otros tantos actores e instituciones. Así, los sujetos construyen una "capacidad estratégica" que los lleva a actuar y pensar en función de los recursos que les rodean y a la vez con cierta autonomía de sí mismos (Lewis y Feinman, 1991; Dubet y Martucelli, 1998). Cuando hay una interiorización del mundo externo, la moral común en el actor social lo obliga a convertirse en su propia referencia y está obligado a tomar sus decisiones. En este proceso de socialización, el individuo adquiere un autocontrol, es decir, reprime sus instintos para crear un mundo íntimo. Aun plenamente socializado, el actor se experimenta a sí mismo como no social, como "propietario de sí mismo". De ahí que la socialización supone la emergencia de un sujeto.

Entendiendo a la educación como un proceso de socialización, se asume la naturaleza social del conocimiento, lo cual desafía su concepción cartesiana. La educación no es el producto de procesos cognoscitivos individuales, sino de prácticas sociales que determinan cómo conocemos, qué es lo que conocemos y qué significado le otorgamos a esos conocimientos. La educación está compuesta de percepciones, significados, intenciones, interacciones, recursos y elecciones, a través de las cuales interpretamos el mundo como sujetos de una época (Sagástegui, 2004).

Desde la psicología cognitiva se ha planteado que el aprendizaje está distribuido y situado, es decir, que no depende de los procesos cerebrales del individuo ni de su capacidad de procesar información, sino que se construye en un marco sociocultural con la intervención de diversos sujetos, a través de mediaciones que se comparten colectivamente, como el lenguaje, los símbolos y las representaciones. Pero además se cuestiona el carácter lineal y conductual del aprendizaje, ya que no se prevé en su totalidad, porque depende del desarrollo de diversas situaciones que no pueden controlarse ni vigilarse.

Finalmente, el conocimiento se basa primordialmente en experiencias (únicas e irrepetibles), ya que son la forma en que la realidad se presenta en nuestra conciencia (Turner y Bruner, 1986). No es la realidad tal cual la percibimos, sino una realidad cargada de juicios razonados, sentimientos y expectativas. Es siempre única y autorreferenciada, es decir, sólo un sujeto puede conocer totalmente sus propias experiencias y éstas constituyen el marco interpretativo de su realidad. A través de las experiencias, los individuos construyen subjetividades, definen su situación presente, elaboran jerarquías y construyen imágenes de sí 
mismos, es decir, definen sus identidades sociales, como profundizaremos en el siguiente apartado.

\section{Cultura, identidad y educación: los aportes del giro intercultural}

Las actuales corrientes cognitivas han puesto los aspectos socioculturales en el centro de las experiencias educativas. Pero situar a la cultura en el centro del aprendizaje no implica necesariamente un cuestionamiento a la posición que ésta tiene en la compleja lucha de fuerzas de todos los campos sociales. ¿Cuáles son las pautas socioculturales que moldean los aprendizajes? ¿Cómo surgen estos entornos generadores de experiencias educativas? ¿Desde dónde se definen estos escenarios socioculturales? ¿Cómo se construyen? Ahora exploraremos el papel de la cultura más allá de los procesos de enseñanza-aprendizaje y de la generación de conocimientos para hablar de las relaciones de poder que enmarcan social y políticamente a las culturas (en plural). En este sentido, el enfoque de la interculturalidad ha puesto a discusión el reconocimiento y la revalorización igualitaria de una diversidad de culturas que, además, se encuentran enmarcadas en relaciones jerárquicas, las cuales explican la dominación de grupos portadores de ciertos aspectos socioculturales sobre otros en la educación tradicional.

Con diferentes tradiciones y matices, la interculturalidad se entiende como aquella que apuesta por un reconocimiento - discursivo y pragmático - de una educación diferenciada para las minorías (Bertely, 2009; Dietz y Mateos, 2011; Mato, 2008). En México ha predominado el enfoque que se plantea la necesidad de multiculturalizar los espacios educativos tradicionales - la institución escolar-, a través de mecanismos de discriminación positiva que permitan empoderar a las minorías, generalmente a las étnicas. Por ello las políticas educativas se dirigen hacia la población indígena de nuestro país, pero no se perciben como una necesidad para las mayorías, lo cual refleja que el multiculturalismo está lejos de ser reconocido como una característica intrínseca y enriquecedora para toda la sociedad. Esto explica en parte que la diferencia cultural siga anclada a los grupos étnicos y no se reconozca en otros sectores (como los juveniles, femeninos o rurales).

Para los académicos y pedagogos aún interesados en el marco de la institución escolar, el enfoque intercultural ha señalado que las políticas integracionistas o asimilacionistas han sido discriminatorias al querer incorporar elementos culturales de los grupos subordinados al espacio escolar, con la intención de lograr una mayor efectividad en los fines universales y homogeneizadores que durante años persiguió la educación moderna. Sin embargo, hoy en día se señala que ya no se trata únicamente de interculturalizar el currículum, sino la praxis 
educativa en sí misma, lo cual implica interculturalizar no sólo el lenguaje y los saberes escolares, sino reconocer (y en muchos casos impulsar) la participación gestiva de los actores locales (mediante un proceso de empoderamiento), a la vez que se promueve la existencia de espacios y formas alternativas para transmitir el conocimiento. De ahí que este enfoque se apropia cada vez más de técnicas de enseñanza no escolares, más participativas y espontáneas (Dietz, 2007; Bertely, 2007).

En México, el fuerte arraigo del indigenismo ha influido decisivamente en cómo se concibe la interculturalidad en el campo de la educación. Por ello estas políticas educativas han transitado por diferentes enfoques, según la concepción que se tenga de los grupos étnicos, aunque las percepciones sobre los indígenas no siempre vayan de la mano con las discusiones académicas. Actualmente, en el ámbito académico se ha cuestionado la perspectiva que consideraba a los grupos étnicos como portadores de una cultura primordial que debía resguardarse para su reproducción. Al parecer, hay cierto consenso en la idea de que los elementos culturales cambian y se resignifican, según los contextos y momentos de interacción entre los indígenas y la sociedad mayoritaria (Barth, 1976); esto nos llevaría a pensar que la interculturalidad en la educación no debe consistir únicamente en la revalorización e incorporación de elementos culturales, como si éstos fueran fijos y no perdieran su valor identitario, sino más bien en la participación autogestiva de los involucrados, especialmente de los beneficiarios. Pero esta gestión desde abajo ocurre una vez que ciertos procesos de afirmación positiva se presentan, ya sea por intervención externa o por medio de procesos de identificación dentro del mismo grupo.

Hasta el momento, la interculturalidad se ha centrado en la población migrante e indígena, pero ha quedado relativamente fuera de la discusión la problemática de otros grupos culturalmente diferentes y subordinados, como la población rural, además de la femenina, los infantes, etc. Hasta ahora se ha discutido el tema del diálogo intercultural con los grupos contrastantes culturalmente hablando, aquellos con los que el choque de prácticas y formas de pensamiento es más intenso, especialmente porque existe el elemento lingüístico de por medio, pero ¿qué pasa con los grupos humanos cuyos referentes culturales no nos parecen tan ajenos, pero no están plenamente reconocidos ni reivindicados? En México hay un importante número de población rural mestiza, especialmente en ciertas regiones, como sucede en el norte del país. Gracias a la población indígena que ha arribado a las ciudades norteñas, aumentando su número de forma exponencial en las últimas décadas, el tema de la interculturalidad se ha comenzado a debatir en términos de política pública -especialmente en el campo educativo-; sin embargo, existe un importante número de población rural mestiza originaria que reside en el campo o que ha migrado desde hace décadas a las capitales y cuyas prácticas y referentes culturales siguen sin ser reconocidos, mucho menos revalorados. Desde el norte, el aporte al debate de la interculturalidad en México sin duda 
se encuentra en esta exigencia de ampliar el panorama de lo que se entiende como culturalmente diverso y dirigir la mirada hacia la población mestiza rural.

En el modelo intercultural, uno de los retos es lograr un diálogo horizontal, una nueva forma de organizar y superar actitudes etnocéntricas, permeadas por una concepción hegemónica de la realidad que se objetiva en la vida cotidiana. Esto nos lleva a reconocer que hay otros conocimientos, formas de comunicación y actores participantes en la transmisión de saberes que trascienden al espacio institucionalizado formal, es decir, a la escuela. Diversas investigaciones en el campo de la educación han señalado la poca efectividad de la escuela entre los sectores rurales, vulnerables, marginados o minoritarios, de ahí que el tema trascienda a los grupos indígenas o migrantes para pensar en las minorías de clase, religiosas, sexuales y de espacios de vida (como las rurales) y la forma en que la educación, en diversos ámbitos vitales, conforma aprendizajes adecuados a sus necesidades y expectativas de vida.

\section{La educación más allá de la escuela: los conocimientos y aprendizajes para la vida}

Uno de los retos más importantes de los proyectos educativos se relaciona con el cumplimiento efectivo de sus fines. El interés por problematizar el papel que desempeña la diversidad cultural, así como los diferentes mecanismos y espacios de socialización que intenta abonar a este reto al evidenciar que existen procesos de enseñanza-aprendizaje significativos, los cuales tienen lugar más allá del espacio escolar institucional, a los que se les debe prestar atención. Por lo general, estos aprendizajes se conocen como "extraescolares", "informales", "incidentales" o "populares" y se les reconoce poca incidencia dentro de la educación formal o institucionalizada.

Para comprender las dinámicas extraescolares, así como el sentido de los aprendizajes obtenidos con éstas, es necesario descentralizar el papel de la escuela para ubicarla como uno más de los campos sociales donde se enseña y aprende significativamente. Nuestra propuesta cuestiona el universalismo prescriptivo que dio origen a la educación moderna, tal como la conocemos ahora. Un cúmulo de investigaciones históricas ha vinculado el origen de la educación institucionalizada con el proceso de conformación de Estados nacionales en Occidente. La ideología liberal fue la que señaló que la educación se convertiría en una de las panaceas del progreso moral, político y económico de las sociedades que aspiraban a ser modernas; para ello la educación tenía que ser laica y controlada por los Estados incipientes, bajo la clara idea de formar a los ciudadanos que las naciones requerían. Varios siglos después, se ha demostrado que, si bien la escuela ha logrado instalarse en lugares recónditos de los países, ha incluido a poblaciones nativas y los maestros se han convertido 
en intermediarios entre los pobladores y el Estado (Arnaut, 1998; Loyo, 1999; Vargas, 1994; González, 2008), lo cierto es que su efectividad aún sigue siendo puesta en duda, debido al carácter homogeneizador y universalista que a lo largo de las décadas ha contado con resistencias y negociaciones locales. La historiografía interesada en los estudios culturales ha logrado reconstruir prácticas y escenarios de interacciones cotidianas, en las que se evidencian las formas y los conocimientos que se transmitían de generación en generación, según las valoraciones sociales de las épocas y los contextos. Este interés por lo cotidiano ha repercutido en las investigaciones históricas de la educación a través del análisis de los objetos para la enseñanza (materiales, manuales), las prácticas disciplinarias, los sistemas de evaluación, la distribución del tiempo y el espacio escolar, las experiencias concretas de mujeres, niños e indígenas, además de otros aspectos visibilizados por estudios micros, alimentados por el método de la etnografía histórica. Sin embargo, la historiografía educativa no ha transitado más allá del espacio de la escuela, sigue circunscrita al espacio institucionalizado, incorporando a la comunidad cuando se enfatizan las resistencias, las negociaciones - las apropiaciones selectivas de las políticas escolares entre los pobladores (Rockwell, 2005). Esta falta de interés en la mirada de los historiadores de la educación provoca que se siga deificando el papel de la institución moderna, que se siga considerando que existe un solo tipo de educación, y que los conocimientos y aprendizajes válidos ocurran en el espacio escolar, principalmente. Esta ausencia se relaciona con un problema de reconocimiento, de valoración positiva y legitimidad de procesos educativos populares, locales y alternativos a la escuela moderna.

Como se señaló antes, ha sido el enfoque intercultural, introducido desde una perspectiva antropológica, el que ha puesto en la mesa del debate el tema de la alteridad, entendida como el reconocimiento del "otro", del "diferente", como miembro de una comunidad y portador de una cultura específica distinta a la que impera de manera dominante. Teniendo la alteridad como principio de inteligibilidad, podemos entender que la educación no se origina ni se limita a la escuela y que la escuela moderna forma parte de un proyecto político de conformación ciudadana que históricamente ha pretendido difundir -y muchas veces imponer- formas de entender el mundo, conocimientos, rituales e identidades específicas que han llegado incluso a deslegitimar otras formas de educación. Al colocar las especificidades socioculturales de un grupo humano en el centro, debiéramos preguntarnos por las formas mediante las cuales un sujeto conoce el mundo que lo rodea y lo aprehende como suyo para conformarse en un ser social, qué tipo de saberes son los apreciados a partir de las formas y expectativas de vida, los mecanismos de transmisión de saberes, los diversos ámbitos en los que se refuerzan y cómo contribuyen a conformar modelos identitarios, a partir de la generación de experiencias significativas que moldean subjetividades. Así, aquello que se ha denominado "conocimiento experiencial" resulta más valorado y útil socialmente. 
Con este enfoque se trata de evidenciar y hacer inteligibles las acciones formativas en sus diversos espacios, formas, agentes, contenidos y temporalidades. Tal como lo señala Buenfil:

Se trata de desnaturalizar los conceptos tradicionales de educación que la reducen a la enseñanza aprendizaje intencionales, socialmente validadas y que ocurren en el ámbito del sistema escolar, para reactivar la multiplicidad de contenidos, estrategias, agentes, espacios sociales y dispositivos mediante los cuales los sujetos se forman, se incorporan a su mundo cultural, se inscriben en el orden simbólico e interactúan con los demás (Buenfil, 2010: 197).

No se trata de negar el papel de la escuela como agente educativo con historicidad propia, sino de descentralizarla de la mirada analítica y complejizar los procesos de enseñanzaaprendizaje, reconociendo la diversidad de contenidos, estrategias, agentes, espacios sociales y dispositivos a través de los cuales los sujetos se forman como seres sociales. La adquisición de capital cultural y social para desenvolverse en el entorno social inmediato ocurre más allá del espacio prescriptivo que ocupa la institución escolar. Si bien es cierto que lo que ocurre cotidianamente en las escuelas escapa del control de las políticas y las autoridades, y que gran parte de los comportamientos y conocimientos se negocian entre los múltiples agentes que participan en este espacio, sigue siendo necesario señalar que la comunidad no sólo está presente en los procesos educativos en los conflictos, las tensiones y las resistencias culturales o políticas que se expresan en su relación con la escuela, sino que es generadora y transmisora de conocimientos que resultan incluso más valorados para las formas de vida social.

En el caso de México, la llegada de la escuela moderna al medio rural ocurrió en las primeras décadas del siglo xx. Fue después de la revolución cuando se llevó a cabo el proyecto de expansión de las escuelas públicas a un importante número de localidades rurales, incluso de difícil acceso. Aun así, las escuelas tuvieron sus limitantes, especialmente en lugares donde la congregación residencial no es una característica demográfica, como ocurre en el extenso territorio norteño; también debido a la falta de recursos materiales para instalar un espacio y mantener al personal, o bien por la falta de interés o la resistencia de la población. Al ser conscientes de este abrupto recorrido en el tiempo, la historiografía de la educación debiera ser más sensible a los procesos educativos extraescolares. Concentrarnos únicamente en la escuela y en la población que confluía alrededor de aquélla es ignorar el mundo de los(as) niños(as) que no asistían por diversos motivos (mujeres en su mayoría); al "otro" tipo de conocimiento que circulaba y era aprendido por la población (me refiero a conocimientos agrícolas, domésticos, de negociación política, rituales religiosos, de comunicación, artísticos, afectivos, etc.); el papel que cumple la familia, el grupo de amigos, los vecinos, el tra- 
bajo o la iglesia en la socialización de los individuos; la transformación de los saberes y las formas de aprenderlos, a partir del ciclo vital de los sujetos, con lo que se evidencia que la educación no termina con la vida escolar; y la diversidad de funciones y finalidades que cada aprendizaje tiene según el ámbito de la vida en la que un sujeto se desenvuelve, sólo por mencionar algunos aspectos. Son procesos educativos que ocurren sin ser previstos, diseñados o promovidos, y que sin duda impactan en la formación de sujetos sociales en sus diversas facetas. Dicho en otras palabras, se trata de colocar en el centro del análisis al individuo y el cúmulo de conocimientos y aprendizajes que forman su vida compleja.

Este interés por la educación fuera de las instituciones formales tiene su origen en los proyectos que se han centrado en la educación de sectores populares, de poblaciones adultas y de grupos indígenas. Desde el enfoque de la "marginación" se vuelven más evidentes las formas de gestión colectiva y el cúmulo de aprendizajes cotidianos que generan experiencias educativas para la vida. Muchas de estas investigaciones provienen del diálogo con posturas vinculadas al pensamiento pedagógico de Paulo Freire. Es en el contexto de las organizaciones políticas populares donde resulta más evidente esta necesidad de reconocer la "otra" educación, así como el valor de los aprendizajes para la vida, porque forman parte de sus reivindicaciones como colectividad. Se trata de perspectivas que en una vertiente más radical terminan en proyectos educativos autonómicos que asumen el derecho a definir y controlar la educación como una lucha contrahegemónica, en resistencia a un sistema que excluye y discrimina los conocimientos, así como las formas de transmitirlo y aprenderlo (Bertely, 2007; Gasché, 2008; Ruiz, 2005).

Pero ¿cómo historiar esta "otra" educación? Como profesionistas de la historia sabemos que el gran reto de nuestras investigaciones se encuentra en la localización y tratamiento interpretativo de las fuentes de información. Para una propuesta transdisciplinaria se requiere una metodología que dialogue con otras fuentes no tradicionales. Hay que señalar que en éstas se requiere de una mayor participación del investigador, quien ya no sólo se debe convertir en una especie de detective detrás de indicios (Ginzburg, 2010), sino en un constructor de expresiones que permitan conocer procesos subjetivos (sentidos, significados, valoraciones, etc.). Estas expresiones se realizan conjuntamente entre el investigador y sus colaboradores -tradicionalmente llamados "informantes"-, a través de narrativas orales, historias de vida, descripciones etnográficas o lecturas del paisaje y los objetos. Para ello es necesario partir de un diálogo presente-pasado, especialmente cuando se trata de interpelar a la "gente sin historia", como diría Wolf (2005), es decir, a los espacios y poblaciones cuyas huellas tangibles son casi inexistentes, debido a su ausencia en los marcos de poder existentes, como ocurre entre las poblaciones rurales del norte y gran parte del territorio mexicano. Esta reconstrucción de sentidos y significados es lo que lleva al historiador a ir detrás de lo "latente", es decir, se trata de mostrar lo que está oculto y que no es observable a primera 
vista (Mendiola, 2006). Lo latente en el pasado se vuelve presente a través de múltiples huellas incompletas que se localizan en fotografías (familiares, de viajes, conmemoraciones escolares, laborales), narrativas (historia oral, epistolares, diarios personales, tarjetas postales, biografías), protocolos (testamentos, actas de bautizo, escrituras, acuerdos), impresos privados (invitaciones, tarjetas), manuales de comportamiento (higiene, economía doméstica, de urbanidad, escolares) y colecciones de objetos (libros, documentos, pintura, impresos). Como toda investigación social, se requiere del cruce de varias fuentes, ya que el ejercicio de reconstrucción histórica nunca es un camino recto y suele ir detrás del dato empírico comprobable.

Para historiarla, toda experiencia de socialización pasada debe trascender al paso de la memoria a través de su objetivación, de su conversión en una huella observable. Para reconstruir la transmisión de formas de entender el mundo se debe ir tras expresiones externas de procesos íntimos, individuales, subjetivos. Y más que estudiar los hechos narrados en sí, se ha de prestar atención a las experiencias, a los sentidos, significados, sentimientos, lógicas de comportamiento presentes en múltiples fuentes, los cuales nunca estarán contenidos en una sola. Para ello se necesita reeducar la mirada, mediante la lectura de indicios, tejiendo relaciones, llenando lagunas y ausencias de significados pasados, así como construyendo nuevas fuentes de investigación a partir de recursos presentes. Se trata de "construir una red de superposiciones y contraposiciones de los trazos en relaciones de analogía, contraste y combinación” (Jetahy, 2007: 367).

Un buen ejemplo de este ejercicio basado en el análisis de procesos socializadores se encuentra en Pablo Piccato (2007), quien estudia, a través de un episodio de protesta popular en la ciudad de México en 1883, cómo los estudiantes ejercen su vida sexual, cómo viven el noviazgo, el consumo de drogas, alcohol y otras prácticas que el autor denomina como la "forma bohemia de ser estudiante". Piccato analiza los aspectos públicos de la identidad de género y cómo se contraponen o complementan con lo privado, es decir, cómo se transmiten y ejercen diferentes formas de ser tanto en espacios públicos, como las escuelas superiores, los barrios pobres donde suelen vivir los estudiantes, las plazas donde se manifiestan, así como en espacios privados (como la familia). Es ese trabajo, Piccato muestra que su condición estudiantil permitía a estos jóvenes explorar la vida capitalina con mayor libertad y atravesar terrenos moralmente prohibidos, a través de los testimonios sobre las prácticas y discursos contenidos en la prensa, los expedientes universitarios, la literatura costumbrista, los libros científicos de la época y las fotografías. En estas últimas hace lecturas interesantes sobre la apariencia corporal de los estudiantes y cómo se van desmejorando a causa de sus excesos bohemios. Sin ser el objetivo central de su trabajo, Piccato muestra cómo los estudiantes se conforman como sujetos sociales a través de prácticas y aprendizajes no sólo vinculados al espacio institucionalizado, sino mediante la 
interacción cotidiana en otros terrenos que les permitían construir referentes identitarios reelaborados en el espacio escolar.

\section{Conclusiones}

Para concluir, señalaré que la propuesta de abrir las investigaciones históricas en educación al análisis de otros espacios y prácticas de formación de individuos permitirá enriquecer, tanto en términos teóricos como metodológicos, los hallazgos de esta disciplina. La intención - como se ha practicado desde hace varios años - no consiste únicamente en retomar los programas, roles y métodos de trabajo, sino captar la manera en que los sujetos construyen su experiencia, estalecen relaciones, estrategias y significaciones, por medio de las cuales se constituyen en sujetos sociales.

Después de conocer exploratoriamente el medio rural neoleonés, potosino y zacatecano, y percatarnos de la excesiva dispersión residencial reflejada en pequeñas localidades con menos de cien pobladores, a lo largo de un extenso territorio semiárido, innegablemente surgen preguntas en torno a las dificultades de acceder a servicios como los educativos en el presente y más aún en el pasado.

Por otro lado, al entender el papel marginal que ocupan y han ocupado históricamente estas poblaciones dentro de las políticas públicas estatales, resulta más evidente la urgencia de generar conocimiento sobre las formas de vida de los habitantes de estas regiones para hacer visibles sus condiciones y necesidades específicas, de manera que se favorezca una intervención política exitosa.

Finalmente, cabe destacar que en el contexto de las intervenciones educativas que combaten la desigualdad y la exclusión social, el extenso campo norteño parece no tener cabida debido a la ausencia de población indígena nativa que reivindique su derecho a otro tipo de educación. A raíz de estas situaciones surge nuestro interés por examinar históricamente los procesos educativos desde perspectivas poco convencionales para la historiografía especializada: desde los procesos de socialización que remarcan la importancia de reconsiderar las experiencias educativas a lo largo de la vida de un sujeto y dentro de otros espacios (como la familia, el barrio, el grupo de amigos, etc.); desde la interculturalidad, con el fin de relativizar el papel deificado de la escuela tradicional y comprender que la diversidad cultural no es exclusiva de los pueblos migrantes ni de los grupos étnicos; así como desde la generación de aprendizajes para la vida, con la intención de otorgarle reconocimiento y validez a las prácticas culturales de poblaciones históricamente desatendidas.

Los alcances y limitaciones de esta propuesta para la historiografía educativa son aspectos que se dirimen en el debate actual, por lo pronto, abrimos la posibilidad de explorar y 
cuestionar las teorías tradicionales, a partir de los hallazgos de nuestras regiones de estudio, con la intención de fortalecer un diálogo transdisciplinario fructífero iniciado desde hace algunas décadas atrás.

\section{Fuentes}

Arnaut, Alberto (1998), La federalización educativa en México, 1889-1994, Secretaría de Educación Pública (SEP), México (Biblioteca para la Actualización del Maestro).

Barth, Fredrik (1976), Los grupos étnicos y sus fronteras. La organización social de las diferencias culturales, FCE, México.

Berger, Peter y Thomas Luckmann (1979), La construcción social de la realidad, Amorrortu, Buenos Aires. Bertely, María (2007), "Educación intercultural para la ciudadanía y la democracia activa y solidaria. Una crítica de la otra educación al multiculturalismo neoliberal y comunitarista”, en Günther Dietz, Rosa Guadalupe Mendoza Zuany y Sergio Téllez Galván (eds.), Multiculturalismo, educación intercultural y derechos indígenas en las Américas, Abya-Yala, Quito, pp. 267-302.

(2009), Sembrando nuestra educación intercultural como derecho: la nueva escuela intercultural en Chiapas, Centro de Investigaciones y Estudios Superiores en Antropología Social (CIESAS)-Alcatraz, México.

Buenfil Burgos, Rosa Nidia (2010), "Dimensiones ético-políticas en educación desde el análisis político del discurso", Revista Electrónica Sinéctica, núm. 35, pp. 1-17.

Chávez González, Mónica (2010), "Familias, escolarización e identidad étnica entre profesionistas tenek y nahuas en la ciudad de San Luis Potosí", tesis de doctorado en Ciencias Sociales, CIESAS Occidente, Guadalajara.

Dietz, Gunther (2007), "La interculturalidad entre el empoderamiento de las minorías y la gestión de la diversidad", Punto de Vista, año III, núm. 12, pp. 27-46.

y Laura Selene Mateos Cortés (2011), Interculturalidad y educación intercultural en México: un análisis de los discursos nacionales e internacionales en su impacto en los modelos educativos mexicanos, SEP, México.

Dubet, François y Danilo Martuccelli (1998), En la escuela. Sociología de la experiencia escolar, Losada, Madrid.

Espinosa Hernández, Armando René (2006), "La institucionalización de la escuela rural federal y la sindicalización del magisterio en San Luis Potosí, 1921-1943", tesis de maestría en Historia, El Colegio de San Luis, San Luis Potosí.

Galván, Luz Elena, Susana Quintanilla y Clara Inés Ramírez (coords.) (2003), Historiografía de la educación en México, Consejo Mexicano de Investigación Educativa (Comie), México (La investigación educativa en México 1992-2002).

Garza, Juana (2010), La educación socialista en Nuevo León, 1934-1940: la atmósfera regiomontana, Facultad de Filosofía y Letras, Universidad Autónoma de Nuevo León, Monterrey.

Gasché, Jorge (2008), "La motivación política de la educación intercultural indígena y sus exigencias pedagógicas: ¿hasta dónde abarca la interculturalidad?", en María Bertely, Jorge Gasché y Rossana Podestá (coords.), Educando en la diversidad cultural: investigaciones y experiencias educativas interculturales y bilingües, Abya-Yala, Quito, pp. 367-397.

Ginzburg, Carlo (2010), Mitos, emblemas, indicios. Morfología e historia, Gedisa, Barcelona. 
González Apodaca, Érika (2008), Los profesionistas indios en la educación intercultural: etnicidad, intermediación y escuela en el territorio mixe, Universidad Autónoma Metropolitana Iztapalapa (UAM, I)-Juan Pablos, México.

Coslin, David A. (ed.) (1969), Handbook of Socialization Theory and Research, Rand McNally and Company, Chicago.

Jetahy, Sandra (2007), "Sensibilidades: escritura y lectura del alma", en Marta Madero y Sandra Gayol(coords.), Formas de historia cultural, Prometeo, Buenos Aires, pp. 361-372.

Lewis, Michael y Saul Feinman (1991), Social Influences and Socialization in Infancy, Plenum Press, Nueva York.

López, Oresta (2010), Que nuestras vidas hablen. Historias de vida de maestras y maestros indígenas tének y nahuas de San Luis Potosí, El Colegio de San Luis, San Luis Potosí.

Loyo, Engracia (1999), Gobiernos revolucionarios y educación popular en México, 1911-1928, El Colegio de México, México.

Mato, Daniel (2008), "No hay saber universal: la colaboración intercultural es imprescindible", Alteridades, vol. 18, núm. 35, enero-junio, pp. 101-116.

Mendiola, Alfonso (2006), Introducción, en Valentina Torres Septién (coord.), Producciones de sentido, vol. 2, Algunos conceptos de la historia cultural, Universidad Iberoamericana, México, pp. 343-356.

Piccato, Pablo (2007), "Sexo, drogas e internado: estudiantes y masculinidad en el México porfiriano", en Marta Madero y Sandra Gayol (eds.), Formas de historia cultural, Prometeo, Los Polvorines, Universidad Nacional de General Sarmiento, Buenos Aires, pp. 137-163.

Ramos Escobar, Norma (2006), "Aspectos profesionales y laborales de la docencia femenina en los procesos de fundación de la educación pública en Nuevo León", tesis de maestría en Historia, El Colegio de San Luis, San Luis Potosí.

(2011), "Concepciones y prácticas de la niñez en la educación pública nuevoleonesa, 1891-1940", tesis de doctorado en Humanidades, Universidad Autónoma Metropolitana Iztapalapa, México.

Rhys Williams, Thomas (1972), Introduction to Socialization. Human Culture Transmitted, Mosby, Saint Louis.

Rockwell, Elsie (2005), "La apropiación, un proceso entre muchos que ocurren en ámbitos escolares", Memoria, Conocimiento y Utopía. Anuario de la Sociedad Mexicana de Historia de la Educación, núm. I, mayo, pp. 28-38.

Ruiz Muñoz, María Mercedes (2005), "El aprendizaje y las prácticas cotidianas en zonas urbanas marginales en la ciudad de México. Casos de estudio", Exploraciones. Revista Interamericana de Educación de Adultos, año 27, núm. 2, julio-diciembre, pp. 71-120.

Sagástegui, Diana (2004), "Una apuesta por la cultura: el aprendizaje situado", Revista Electrónica Sinéctica, núm. 24, febrero-julio, pp. 30-39.

Turner, Victor y Edward M. Bruner (1986), The Anthropology of Experience, University of Illinois Press, Chicago.

Vargas, María Eugenia (1994), Educación e ideología. Constitución de una categoría de intermediarios en la comunicación interétnica. El caso de los maestros bilingües tarascos (1964-1982), CIESAS, México.

Whiting, John, Irving Child y William Lambert (1966), Field Guide for a Study of Socialization, vol. 1, Harvard University, John Wiley and Sons (Six Culture Series).

Wolf, Eric (2005), Europa y la gente sin historia, FCE, México. 
Mónica Lizbeth Chávez González. Escuela Nacional de Estudios Superiores, Unidad Morelia, unam. Doctora en Ciencias Sociales, especializada en Historia y Antropología de la Educación, Género y Etnicidad. Publicaciones recientes: Identidad étnica, migración y socialización urbana. Profesionistas indígenas de la Huasteca en la capital potosina, CIESAS (en prensa) y de "La familia, las relaciones afectivas y la identidad étnica entre indígenas migrantes urbanos en San Luis Potosi”, en Relaciones 135, vol. 34 (primavera) Zamora, El Colegio de Michoacán, 2013, pp. 131-155.

Recibido: 22 de marzo de 2013

Aceptado: 30 de julio de 2013 